\section{Next Step in Sight for Gravity Waves}

Theory predicts that the oscillating curvature of a gravitational wave generated by various astrophysical events acts as a tidal force to induce oscillations in the separation between two neighbouring test particles. Differential displacements (in the $10-100 \mathrm{~Hz}$ to $5 \mathrm{kHz}$ frequency range) would be produced in the arms of a long-baseline, free-mass, laser interferometer. The 210 MSUS LIGO project in the US involves the construction and operation by 1998 of the first km-scale interferometer observatory comprising two facilities each with $4 \mathrm{~km} \times 4 \mathrm{~km}$ interferometers (budget allocations have so far been close to planned levels).

The other detection technique uses the dynamic strain induced in a resonant mass. In a typical bar-type antenna, a transducer measures excitation of the fundamental longitudinal mode of a solid aluminium cylinder with a resonance near $1 \mathrm{kHz}$. Low-noise, cryogenic, resonant-mass detectors were first proposed in 1960. They are modest in scale, fairly reliable, but limited in bandwidth and having in principle a strain sensitivity $h$ at the quantum limit which is $\mathbf{5 0}$ times less than for a large interferometer. Following early setbacks, pioneers at Stanford achieved in 1981 an $h$ of $10^{-18}$ with a $5 \mathrm{t}$ bar at $4.2 \mathrm{~K}$. Similar devices (ALLEGRO at Louisiana State University and the University of Rome's EXPLORER detector at CERN) were operated soon after.

Plans for third-generation bar antenna cooled to $50 \mathrm{mK}$ were being prepared at the same time and a progress workshop (Cryogenic Gravitational Wave Antennae, INFN, Legnaro, 19-23 June 1993) showed that these efforts are close to culmination. The NAUTILUS detector at INFN's Frascati laboratory has a planned sensitivity of $2 \times 10^{-19}$ (compared with $6 \times 10^{-19}$ for the $4.2 \mathrm{~K}$ devices). Cryogenic testing to $100 \mathrm{mK}$ finished in January and datataking should start this summer. A similar device called AURIGA (2.3 t Al alloy bar; resonant capacitor strain measuring transducer; 915 $\mathrm{Hz}$ ) has been assembled at the INFN Legnaro laboratory ready for the first cool-down this autumn. Stanford University will begin cooling down later this year its $2.2 \mathrm{~m}$ long bar antenna. The strain sensitivity will initially be $\approx 10^{-19}$ but at least $10^{-20}$ is reported to be attainable, and levels approaching $3 \times 10^{-21}$ (close to the quantum limit) should be possible with thin-film superconducting transducers under development. These three ultralow-temperature antennae will be working in coincidence for most of the 1990's looking for rare sources in our galaxy (the estimated event rate increases from about one every 10 years to maybe a few events in roughly the same period as the sensitivity increases to $3 \times 10^{-21}$ ).
It is planned to continue datataking with the $4.2 \mathrm{~K}$ devices, notably ALLEGRO and EXPLORER (which had to abort its last run starting in January because a new transducer gave an unexpected poor sensitivity) Following modifications to the insulation structure, a group at the University of Western Australia, Perth, had to stop the first attempt in May to cool its $1.5 \mathrm{t} \mathrm{Nb}, 700 \mathrm{~Hz}$ detector to $4.2 \mathrm{~K}$ in order to relocate a pump. Meanwhile, a $4.2 \mathrm{~K}, 1.2 \mathrm{kHz}$ torsion-type detector with a $1.7 \mathrm{t} \mathrm{Al}$ alloy disc offering an estimated $h$ of $2 \times 10^{-19}$ is under construction at Tokyo University.

The resonant detector community, aside from developing its instruments, must address several other important issues, including what is meant by experimental evidence for a gravity wave. Guido Pizzella of the University of Rome who has masterminded the Italian effort for many years (his 60th birthday was celebrated at the Legnaro meeting) argues that the search for coincidences remains "the final and most valid means". But it may not simply be sufficient to establish that the probability of a coincidence occurring by chance is small. The next five-year INFN budget proposal, which should be approved by the Italian government this autumn, includes support for two more detectors of the NAUTILUS/AURIGA type to create a true observatory.

Cosmic rays produce signals in resonating detectors. Hadrons, the dominant source, are easily shielded out, but the smaller muon contribution requires a fairly thick overburden. Fourth-generation generation detectors seeking strain sensitivities of $\approx 10^{-21}$ will have to tackle such issues. As it is thought difficult to reduce the noise temperature to much below today's $\approx 7 \mathrm{mK}$, ideas for these devices centre on large cross-section, omnidirectional, spherical or near-spherical antennae costing roughly twice as much (10-20 MSUS) as $50 \mathrm{mK}$ antennae. Designs speak of 20-40 $\mathrm{t} \mathrm{Al}$ alloy spheres cooled in a vacuum to below $100 \mathrm{mK}$ inside $8 \mathrm{~m}$ large cryostats and having five superconducting transducers. The Louisiana (LSU) group estimates that its TIGA design based on a $2.6 \mathrm{~m}$ in diameter, $25 \mathrm{t}, 1 \mathrm{kHz}$, truncated $\mathrm{Al}$ alloy icosahedron would be 56 times more sensitive than the equvalent bar antenna with the same noise temperature, thus bringing the "assured" event rate to a few per year. Such devices were proposed more than 20 years and several spherical geometries have been investigated at LSU, notably a $0.9 \mathrm{~m}$ truncated icosahedron set up last winter.

G. Frossati, well known for contributions to low-temperature condensed-matter physics (see page 108), working with the Rome group described how a $30 \mathrm{t}$ spherical detector (see figure) could be cooled to $10 \mathrm{mK}$ in a reasonable time using two dilution refrigerators about twice as large as those available today. One option before tackling the construction of such a device would be to set up a network of smaller spherical antenna.

The development of room-temperature antennae with sensitivities of a few times $10^{-18}$ is not being abandoned as they can be left unattended for long periods of observation. The latest plans include a fourth detector in the CRAB series (CRAB IV: $2 \mathrm{~m}$ in diameter; $1.7 \mathrm{t} ; 1.2 \mathrm{kHz}$; Al alloy disc) being constructed at KEK, Tsukuba. As the sensitivity is some five times less than that needed to detect gravity waves from the Crab pulsar, there is interest in monitoring the recently discovered PSR 10437 pulsar which would give a stronger effect, but at a lower frequency.

A group at the Sternberg Astronomical Institute, Moscow State University, led by V.N. Rudenko has been able to maintain part of the former Soviet Union's "five directions" astrophysical observation programme etablished in the 1980's. Plans to complete a large cryogenic bar detected are shelved but a $1 \mathrm{t}$ $2 \mathrm{~m}$ room-temperature antenna (called Snail owing to its unusual shape) is ready for longterm observations at sensitivities of $7 \times 10^{-17}$ (improvements may raise $h$ to about $10^{-18}$ ). The hope is that Snail could be sponsored as part of a global network since only token support is received from the Russian Academy of Sciences to supplement modest support from Russia's nuclear physics programme. The Moscow group is also developing transducers - an extremely active field as quantum nondemolition techniques [EN 24 (1993) 51] will be needed to push $h$ beyond the standard quantum limit.

But perhaps the most pressing needs are to coordinate efforts within the worldwide cryogenic detector community and to ensure complementarity with $\mathrm{km}$-scale interferometers, notably LIGO in the USA, the VIRGO detector proposed by the INFN and the French CNRS [EN 23 (1992) 217], and possibly the proposed $3 \mathrm{~km} \times 3 \mathrm{~km}$ AlGO in Australia. Indeed, a statement along these lines was issued at the workshop. 